\title{
Role of granule proteases in the life and death of neutrophils
}

Charaf Benarafa ${ }^{1^{*}}$, Hans-Uwe Simon ${ }^{2^{\star}}$ 


\section{Abstract}

Neutrophils constitute a crucial component of the innate immune defenses against microbes. Produced in the bone marrow and patrolling in blood vessels, neutrophils are recruited to injured tissues and are immediately active to contain pathogen invasion. Neutrophils undergo programmed cell death by multiple, context-specific pathways, which have consequences on immunopathology and disease outcome. Studies in the last decade indicate additional functions for neutrophils - or a subset of neutrophils - in modulating adaptive responses and tumor progression. Neutrophil granules contain abundant amounts of various proteases, which are directly implicated in protective and pathogenic functions of neutrophils. It now emerges that neutral serine proteases such as cathepsin $G$ and proteinase-3 also contribute to the neutrophil life cycle, but do so via different pathways than that of the aspartate protease cathepsin $D$ and that of mutants of the serine protease elastase. The aim of this review is to appraise the present knowledge of the function of neutrophil granule proteases and their inhibitors in neutrophil cell death, and to integrate these findings in the current understandings of neutrophil life cycle and programmed cell death pathways. 


\section{Specificities of neutrophil life and death}

Granulopoiesis and neutrophil lifespan. The role of neutrophils in antimicrobial surveillance is undisputed as quantitative and qualitative defects in neutrophils lead to severe immunodeficiency [1]. Additional functions for neutrophils in immunomodulation have been described in certain disease contexts indicating that a small subset of neutrophils contribute to adaptive immune responses (see reviews [2-4]). These functions suggest that neutrophils are not homogenous. Seminal studies in the 1950s and 1960s in humans and various laboratory animals have shown that neutrophils labeled ex vivo and re-injected, or directly labeled in vivo have a short circulatory lifespan of less than a day [5-8]. These findings have remained unchallenged until an average lifespan of more than 5 days for human neutrophils was reported using a novel, non-toxic tracer [9]. The study received a lot of attention and, in the meantime, over 150 citations. The appeal of these findings is understandable: the experimental work is sound and the conclusions are provocative as they suggest that homeostatic neutrophil lifespan is sufficiently long to allow neutrophils to execute novel functions such as interaction with antigen-presenting cells and lymphocytes in the context of adaptive immune responses and cancer.

So was our textbook knowledge wrong regarding the neutrophil lifespan? Neutrophils derive from hematopoietic stem cells (HSCs) and develop in the bone marrow through a common myeloid progenitor shared with the monocyte/macrophage lineage. The process of granulopoiesis takes committed neutrophil progenitors from the stem cell pool and brings them through differentiation into mature neutrophils that then enter the blood circulation. These precursors first go through a proliferative phase called the mitotic pool followed by a non-proliferative phase named the post-mitotic pool, where cells further differentiate without dividing. Based on cellular size, granule content and nuclear morphology, several neutrophil differentiation stages were identified. The mitotic pool includes myeloblasts, promyelocytes and myelocytes, while metamyelocytes, band and mature neutrophils constitute the post-mitotic pool. To determine neutrophil lifespan in vivo, one can label neutrophils ex vivo and reinject them to measure the kinetics of cell disappearance from the blood circulation. This approach is straightforward but has important caveats including neutrophil activation during ex vivo manipulation and toxicity of the labeling method or compound. In vivo labeling has thus been performed with a pulse of tritiated $\left({ }^{3} \mathrm{H}\right)$-thymidine to label the DNA of cells in the mitotic pool or by using ${ }^{3} \mathrm{H}$ or ${ }^{32} \mathrm{P}$-labeled diisopropyl fluorophosphate (DFP), which irreversibly reacts with the catalytic site of neutrophil serine proteases. These radioactive compounds are not available anymore for clinical research because of their inherent toxicity. Pillay et al. performed their study by labeling proliferating bone marrow cells in vivo by giving human subjects and mice deuterium labeled "heavy" water $\left({ }^{2} \mathrm{H}_{2} \mathrm{O}\right)$, a safe and nonradioactive 
tracer that can later be detected in adenosine in the DNA of circulating neutrophils by gas chromatography coupled with mass spectrometry [9]. They first examined both bone marrow and blood neutrophil kinetics of mice and found that mouse neutrophils have a short lifespan of about 18 hours as shown previously. They then calculated that circulating neutrophil lifespan is at least 5 days in human subjects following a 9-week labeling period with heavy water, a value perhaps 10 -fold greater than that gleaned from long standing studies using toxic tracers and/or ex vivo labeling.

Calculating neutrophil lifespan in humans is not as easy as in mice because sampling the bone marrow compartment is highly invasive and is not available to investigators for repeated sampling. Moreover, modeling human neutrophil lifespan has to be calculated based on several assumptions regarding proliferation rate of the mitotic pool, transit time from the last proliferation to entry in the blood circulation, apoptosis within the bone marrow and determine neutrophil numbers based on ratios of labeled vs non-labeled adenosine. Indeed, using the same data but assuming that the mitotic pool is the ratelimiting factor, Dale and colleagues suggested that the data was actually compatible with a neutrophil lifespan of less than a day [10]. A new study now appears to settle the issue using ${ }^{2} \mathrm{H}$-labeled glucose in parallel with a limited set using double-labeling with heavy water [11]. ${ }^{2} \mathrm{H}$-glucose is more efficiently and rapidly metabolized in adenosine to be incorporated into the DNA of the mitotic pool, allowing labeling over a few hours or in a single injected bolus instead of weeks for heavy water and therefore more precise estimates of neutrophil half-life. Using a new model, they found that different assumptions of the ratio $(R)$ of circulating cells to the mitotic pool is the key factor that can predict neutrophil lifespan of 1 or 5 days. The numbers suggest that the mitotic pool is five times larger than the circulating pool with $\mathrm{R}=0.2$. The model of Koenderman and colleagues [9] leading to a long neutrophil lifespan implies a large value of $R$ that is not compatible with historical measurements of the various neutrophil compartments. So it seems that the old studies had it right, at least until a new method, tracer or model will again naturally challenge our brains and dogmas.

Cell death in neutrophils. Programmed cell death (PCD) can be executed by various cell autonomous mechanisms that are highly intertwined and triggered by both internal and external signals (Figure 1) [12]. Apoptosis is the best molecularly characterized mode of cell death and it culminates in the activation of a subset of cysteine proteases with aspartate specificity, known as caspases, which irreversibly execute cell death through cleavage of multiple targets in the nucleus, cytoplasm and cytoskeleton. Apoptosis has been recognized as the main PCD pathway that is triggered upon isolation of blood neutrophils and is often referred to as "spontaneous" or "constitutive" apoptosis. PCD was 
until recently synonymous to apoptosis, while necrosis did not require any active mechanistic input from the dying cell and resulted from external mechanical, physical, chemical and osmotic stresses causing the rupture of the plasma membrane and release of the cytosolic contents. It is now accepted that necrosis can also result from distinct molecular pathways that have been defined as necroptosis and pyroptosis, which, unlike apoptosis, lead to cell membrane lysis [13]. Necroptosis generally proceeds when caspases (particularly caspase-8) are inhibited or inactive and is triggered by specific protein kinases, principally receptor-interacting protein kinase-3 (RIPK3). Ultimately, necroptosis was shown to be dependent on the pseudokinase mixed lineage kinase domain-like protein (MLKL), which is thought to have pore-forming capacity [14]. Necroptosis has recently been shown to occur in neutrophils following phagocytosis of Staphylococcus aureus [15] and ligation of adhesion receptors [16]. Pyroptosis depends on the cleavage of the cytoplasmic gasdermin $D$ by caspase- $1,-4,-5$ and -11 following inflammasome activation [17-19]. However, pyroptosis appears so far not to be evoked in neutrophils [20]. Secondary necrosis is readily observed in neutrophils in vitro when isolated neutrophils are left to undergo spontaneous apoptosis and, after some time, their plasma membrane becomes permeable and they release their cytoplasmic content. Secondary necrosis also occurs in vivo when phagocytic removal of apoptotic cells is impaired or overwhelmed [21].

PCD pathways associated with neutrophil granule functions are incompletely defined. Neutrophils are exquisitely sensitive to cell death induced by lysosomotropic agents, which disrupt granule/lysosome membranes and lead to PCD with features of both apoptosis and necrosis [22,23]. Neutrophil PCD can also occur concurrently with autophagy and phagocytosis, where granules fuse with other vesicular compartments $[24,25]$. "NETosis" was described as a form of neutrophil death associated with the release of neutrophil extracellular traps (NETs) composed of DNA and granule proteins [26]. While DNA release is documented to occur in vivo in the context of infections and inflammatory diseases [27-29], it is debated whether this is necessarily caused by neutrophil death $[30,31]$. Indeed, NETosis may be a form of cell death that is principally an in vitro phenomenon [32].

Positive and negative feedbacks of neutrophil cell death. The mode of cell death has consequences on the surrounding environment and inflammatory responses. This is especially relevant for neutrophils, which contain granules filled with reactive chemicals and enzymes [33]. Apoptosis is thought to have a neutral or even anti-inflammatory impact when it is followed by efferocytosis, which is the swift removal of the dead cells by phagocytes or neighboring cells [21,34]. In steady state, aging neutrophils are removed 
by macrophages in the bone marrow, spleen and liver [35]. In addition to antiinflammatory effects, efficient efferocytosis prevents damage associated with secondary necrosis and reduces risks of autoimmune disease [36-38]. Importantly, phagocytosis of apoptotic neutrophils reduces the constitutive production of IL-23 by macrophages, which in turn reduces the production of $\mathrm{IL}-17$ and G-CSF as a negative feedback on granulopoiesis [39]. Therefore, the efficient removal of apoptotic neutrophils is a key regulatory loop that prevents tissue damage associated with secondary necrosis and ultimately regulates neutrophil production in the bone marrow. Necrosis can have both beneficial and destructive effects depending on the magnitude of the reaction, its location and the cause of the trigger. For example, danger associated molecular patterns (DAMPs) associated with limited neutrophil necrosis may induce positive feedback loops to ameliorate both innate and adaptive immune responses. It was recently shown that necrotic cell death may even help trap phagocytosed pathogens despite plasma membrane rupture [40]. In most cases however, necrosis observed after massive and sustained neutrophil recruitment such as in cystic fibrosis or chronic obstructive pulmonary disease is a significant driver of pathology [37]. Understanding mechanisms of PCD provide specific targets for therapeutic modulation in infectious, inflammatory and autoimmune diseases.

\section{Effects of neutrophil proteases on neutrophil life cycle}

Neutrophil granule production. Neutrophils contain many cytosolic granules that are lysosome-related organelles containing a wide range of soluble and transmembrane proteins that contribute to antimicrobial functions, immunomodulation, extracellular matrix remodeling and, as discussed below, cell death. The types of neutrophil granules and secretory vesicles have been described based on protein content, cytochemical staining properties and electron microscopy features. These organelles are sequentially produced during neutrophil terminal differentiation from the promyelocyte stage up to the segmented mature neutrophil. The targeting of proteins to various granule types is well correlated with transcriptional timing during neutrophil differentiation indicating that expression rather than protein sorting is the main driver of granule contents [41]. Primary (azurophil) granules, rich in myeloperoxidase and serine proteases, are only produced at the promyelocyte stage. At the myelocyte stage, secondary (specific) granules are then produced and are characterized by higher content in lactoferrin and neutrophil gelatinaseassociated lipocalin (NGAL). In post-mitotic stages of differentiation, tertiary (gelatinase) granules expressing high levels of matrix metalloproteinase 9 (MMP9) and ficolin are produced [42]. Neutrophils also contain secretory vesicles, which appear from the band 
stage and are thought to be produced by endocytosis of the plasma membrane as they contain serum proteins.

Proteomic analyses of neutrophil granule contents following density gradient separation indicate that proteins are generally not restricted to a single granule type but instead show a certain spectrum of distribution [43,44]. While there is a good correlation between the timing of mRNA expression and proteome of various granule types, it is not entirely straightforward and discrepancies have been found [43]. Additional mechanisms such as sorting, miRNA regulation, post-translational processing are likely to explain differences and determine whether a particular protein will be found in a single or multiple types of granules. For example, serglycin is required for the sorting of neutrophil elastase (NE) to primary granules, while it is not required for cathepsin G (CatG) although they both end up in primary granules [45]. Localization of soluble and membrane proteins to various types of granules is of importance because stimuli required to induce fusion of each organelle with the plasma membrane or the phagosome is not equal. Secretory vesicles and ficolin-rich granules more readily fuse with the cell membrane than gelatinase and specific granules. Primary granules are only mobilized to higher stimulations; they are the last to be secreted and have a preference for fusing with the phagosome.

Because they are produced at the promyelocyte stage, primary granules are then divided between daughter cells during cell division and therefore they are much more abundant in promyelocytes than in the post-mitotic pool and circulating neutrophils. Of note, studies of granule contents have been performed during steady state granulopoiesis. Further work is needed to investigate the granule composition of neutrophils produced during emergency granulopoiesis associated with severe infections, chronic inflammatory diseases and sepsis. Indeed, the protein cargo, the number of different types of granules, and their degranulation propensity may be altered by changes in transcription, number of mitotic divisions, shortened post-mitotic maturation time and protein sorting in disease contexts.

Neutrophil granule proteases. Granules contain several proteases of the serine, cysteine, aspartate and metallo-protease families. NE, CatG and proteinase-3 (PR3) are the three best characterized and most abundant serine proteases of neutrophils [46]. Their expression pattern is similar to that of myeloperoxidase and they are thus principally found in primary granules. Yet, PR3 has a plasma membrane-associated form that is also found in secretory vesicles [47]. Neutrophil serine protease-4 (NSP4) and cathepsin A are also serine proteases principally found in primary and tertiary granules, respectively. Azurocidin is encoded by a gene phylogenetically related to NE and is found in primary 
granules but its catalytic domain is mutated rendering the protease inactive. CatD and CatE are aspartate proteases, and cathepsins $\mathrm{B}, \mathrm{H}, \mathrm{K}, \mathrm{L}, \mathrm{S}, \mathrm{Z}$ are cysteine proteases that are found in all types of granules but in relatively higher abundance in tertiary and ficolinrich granules [43]. Cathepsin C, also a cysteine protease and better known as dipeptidyl peptidase I (DPPI), is found at highest levels in primary granules. DPPI is an aminodipeptidase that is required for the activation of all neutrophil serine proteases by the removal of an N-terminal pro-dipeptide [48]. The matrix metalloproteinase (MMP) gelatinase B (MMP9) is found at highest levels in tertiary granules, which are also named for that reason gelatinase granules. MMP9 is also found at lower levels in secondary granules. Leukolysin (MMP25), a membrane-anchored protease, and matrilysin (MMP8) are both found principally in tertiary granules. In contrast to serine and cysteine proteases, MMPs are stored as zymogens within granules and require cleavage of a propeptide domain after degranulation to become active [49].

Serpinb1: a major neutrophil survival factor in vivo. Neutrophil protease activity following release in the extracellular milieu is regulated by plasma protease inhibitors produced by hepatocytes as well as locally produced inhibitors in tissues and epithelial surfaces. While neutrophils do not express tissue inhibitors of metalloproteases (TIMPs), they express several inhibitors of the serpin family that inhibit neutrophil granule serine and cysteine proteases. Unlike most other types of inhibitors, which use a key-lock mechanism of inhibition, serpins are fast suicide-substrate inhibitors that make covalent complexes with their target proteases with a 1:1 stoichiometry [50]. Among them, serpinb1 has emerged as a key regulator of neutrophil survival. Serpinb1, previously known as MNEI (monocyte/neutrophil elastase inhibitor) or LEI (leukocyte elastase inhibitor), belongs to the clade B serpin family, which is composed of non-glycosylated 40$45 \mathrm{kDa}$ proteins without cleavable signal peptide [51,52]. Serpinb1 has a nucleocytoplasmic localization and inhibits CatG, NE and PR3. Knockout mice of serpinb1a, the mouse orthologue of the human gene [53], have a $50 \%$ reduction in mature neutrophil numbers in the bone marrow and are susceptible to $P$. aeruginosa infections [54,55]. This reduction is highly relevant since, in mice, the bone marrow holds $90 \%$ of mature neutrophils.

The mechanism of cell death in the absence of serpinb1a is dependent on CatG. Indeed, neutropenia in serpinb1 $\mathrm{a}^{-/-}$mice is fully rescued by deficiency in CatG. In contrast, NE deficiency had no effect on neutrophil survival in serpinb1a ${ }^{-/}$mice [56]. The cytoprotective mechanism of serpinb1 in neutrophils and the CatG-mediated death is cell autonomous as demonstrated by transgenic rescue with human serpinb1 in mouse neutrophils, conditional deletion in myeloid cells, and competitive bone marrow chimera 
$[56,57]$. Importantly, mice lacking NE, CatG, PR3 or all serine/cysteine proteases (as in $\mathrm{dppi}^{--}$mice) do not present increased neutrophil numbers in bone marrow and blood [56,58-61].

While serpinb1 appears as a principal component of the antiprotease shield, it is likely that other inhibitors protect neutrophils against granule proteases. Characterization of mice deficient in multiple intracellular serpins may reveal complementary as well as independent functions. In particular, serpinb6, also known as protease inhibitor 6 (PI-6), is expressed in the cytoplasm of neutrophils and monocytes and inhibits CatG [62]. Mice deficient in serpinb6a, the orthologue of the human gene [63], do not present a defect in neutrophil numbers in vivo but expression of serpinb1a is increased in neutrophils of serpinb6a $^{-/}$mice [64]. Serpina3g (also known as Spi2A) is a mouse clade A serpin which has homology with human $\alpha 1$-antichymotrypsin (SERPINA3). Serpina3g is highly atypical for a clade A serpin because it lacks a cleavable signal peptide and has a nucleocytoplasmic localization and thus its functions may resemble more those of clade $B$ serpins [65]. Of note, there are no human equivalent of Serpina3g: human antichymotrypsin is encoded by a single gene, whereas the mouse locus includes 14 serpina3 paralogues [66]. Serpina3g inhibits several cysteine proteases including CatB and appears to regulate lysosomal-induced cell death induced by CatB in various cell types including hematopoietic stem cells but deficiency in serpina3g is not associated with reduced neutrophil numbers $[67,68]$. These findings indicate that neutrophil homeostasis in steady state depends on intracellular inhibitors, which prevent or delay apoptotic death mediated by granule proteases.

Serine and cysteine proteases in neutrophil apoptosis in vitro. Mechanisms regulating neutrophil cell death have been principally investigated in vitro, where isolated blood neutrophils rapidly undergo characteristic apoptotic death in the absence of a known trigger (Figure 1) [12,69]. This process is often referred to as spontaneous or constitutive apoptosis and is mechanistically dependent on cytoplasmic activation of effector caspases with classical features of apoptosis such as mitochondrial outer membrane permeabilisation (MOMP), release of cytochrome c, nuclear condensation, cell shrinking, membrane blebbing, and phophatidylcholine exposure on the outer leaflet of the plasma membrane [70]. Despite having fewer mitochondria and relatively low amounts of cytochrome $c$, the mitochondrial or intrinsic pathway of apoptosis is key to neutrophil constitutive apoptosis $[71,72]$. Bcl-2 family proteins regulate the integrity of the outer mitochondrial membrane. Mature neutrophils do not express Bcl-2 but rely on Mcl-1 and $\mathrm{A} 1 / \mathrm{Bfl}-1$ as anti-apoptotic $\mathrm{Bcl}-2$ family proteins to bind pro-apoptotic $\mathrm{Bcl}-2$ proteins and prevent MOMP triggered by Bax and Bak. BH3-only proteins constitute the third 
group of Bcl-2 family proteins such as Bid, Bim, and Noxa, which are also pro-apoptotic and provide an additional layer of regulation by sequestering anti-apoptotic proteins in heterodimers and/or by enhancing the activity of pro-apoptotic Bak and Bax. MOMP leads to the release of several compounds including cytochrome c, Smac/Diablo and the serine protease HtrA2/Omi, which interact with cytosolic inhibitors of apoptosis (IAPs) and promote the assembly of the apoptosome.

Cell cycle regulatory proteins were recently found to contribute to neutrophil survival, a surprising finding since mature neutrophils are non-proliferating cells $[73,74]$. As its name suggests, proliferating cell nuclear antigen (PCNA) was originally reported to be associated with the activity of DNA polymerases in the nucleus of actively replicating cells including neutrophil progenitors. However, the trimeric form of PCNA was also found to be expressed exclusively in the cytoplasm of blood neutrophils and was found at higher levels in mature neutrophils of patients treated with G-CSF. PCNA appears to interact with many proteins [75] and it is thought that PCNA blocks neutrophil apoptosis by sequestering pro-caspases [76]. Most intriguingly, PCNA is known to interact with cyclin dependent kinases (CDKs) as well as the endogenous CDK inhibitor p21/waf1. Treatment of neutrophils with R-roscovitine, an inhibitor of CDKs expressed at low levels in mature neutrophils, induces neutrophil apoptosis [77]. Moreover, a p21-derived peptide known to inhibit cell proliferation by interacting with PCNA in replicating cells was shown to trigger neutrophil apoptosis, likely by disrupting interactions proteins bound to PCNA [76]. Of note, p21/waf1 was previously shown to be cleaved by PR3, which positively regulated the proliferation granulocyte progenitor cell lines [78]. Proteolytic regulation of PCNA or its bound partners may also contribute to neutrophil PCD.

Cytoplasmic release of granule/lysosome cathepsins leads to apoptosis through the mitochondria or by inactivation of IAPs [79]. CatD, but not CatB, was shown to induce neutrophil apoptosis through direct cleavage of caspase-8 in human and mouse neutrophils $[80,81]$. Caspase-8 activation induces apoptosis through direct activation of effector caspases-3, -6 and -7 . In addition, caspase-8 activates the mitochondrial pathway, which was shown in mice to be mediated by the cleavage of Bid into its active form tBid. PR3-deficient neutrophils also show delayed apoptosis in vitro compared to wild-type neutrophils and PR3 is found in the cytoplasm of aging neutrophils in vitro. Inhibitory complexes between PR3 and serpinb1 are found in the cytoplasm of aging neutrophils. PR3 directly cleave pro-caspase-3 leading to apoptosis [61]. Conversely, serpinb1a $^{-/-}$neutrophils also demonstrate reduced survival in vitro that is only partly delayed by caspase inhibitors [56]. In the presence of caspase inhibitor Q-VD-Oph, the survival defect of serpinb1 $\mathrm{a}^{-/}$neutrophils is fully rescued if neutrophils are concomitantly deficient in serpinb1a and CatG. As described above for the in vivo situation, neutrophils 
lacking NE and serpinb1a show fast kinetics of cell death, indicating no role for NE in this form of cell death. Therefore, CatG induces neutrophil death through both caspasedependent and independent pathways when serpinb1 levels are low. While CatG has been shown to process pro-caspase-7 into its active form [82], the targets of CatG leading to caspase-independent cell death are not yet know. Overall, serpinb1 inhibits spontaneous apoptosis mediated by both CatG and PR3. It is conceivable that CatG, PR3 and even CatD function in a cascade of sequential proteolysis because CatG deletion alone is sufficient to rescue the cell intrinsic death pathway in serpinb1 $\mathrm{a}^{-/}$neutrophils in vitro and in steady state conditions in vivo. More likely, these proteases induce neutrophil death through partly overlapping pathways that would enhance each other. Recently, serpinb1 was shown to inhibit granzyme $\mathrm{H}$ as well as cysteine cathepins $\mathrm{CatH}$, CatS and to a lesser degree CatL, CatV and CatK but not CatB, CatC and CatK $[83,84]$. The significance of these interactions in neutrophil survival remain to be investigated.

Elastase mutations and neutropenia. Studies of NE knock-out mice and in combination with serpinb1 deficiency indicate that NE has no effect on neutrophil survival and cell death induced by granule leakage $[56,58]$. Nevertheless, close to two hundred distinct mutations in ELANE (the gene encoding NE) have been identified as the cause of severe congenital neutropenia (SCN) $[85,86]$. SCN is a rare, genetic disease characterized by persistently low circulating neutrophil counts $(<500 / \mu \mathrm{l})$ and a maturation arrest at the promyelocyte stage in the bone marrow. From a very young age, SCN patients develop multiple infections that can be fatal. Autosomal dominant mutations in ELANE are the cause of $>50 \%$ of SCN cases and of all cases of cyclic neutropenia (CyN) $[87,88]$. CyN is a milder form of SCN where blood neutrophil counts oscillate between normal and very low counts with a cyclic period of 21 days [89]. Other genetic causes have been identified such as autosomal recessive mutations in the HAX1 (HCLS1-associated protein X-1) gene, the Jagunal homolog 1 (JAGN1) gene and, more rarely, by mutations in the adenylate kinase 2 (AK2), the transcriptional repressor (GF/1) and the glucose-6phosphatase (G6PC3) genes [90-92]. A subset of patients with Wiskott-Aldrich syndrome were also shown to develop X-linked neutropenia [93]. Importantly, many SCN cases remain genetically undefined but advances in sequencing technology will likely unveil new genes and mechanisms causing this primary immunodeficiency.

The current working paradigm regarding mutant NE and neutropenia is that apoptosis is induced at the promyelocyte/myelocyte stage in the bone marrow via the initiation of the unfolded protein response (UPR) [94,95]. NE mutants are predicted to have defective folding as NE transcription/translation surges in promyelocytes. ER sensors detect misfolded NE and trigger the UPR that can ultimately lead to cell death. 
Genotype-phenotype analysis studies suggest that the pattern of mutations in ELANE is different in patients that develop SCN or CyN [86]. Yet, some mutations lead to both SCN and CyN indicating that additional disease modifiers may impact the phenotype [85]. Interestingly, Skokowa and colleagues found low levels of secretory leukocyte protease inhibitor (SLPI) in SCN but not CyN patients [96]. They then showed that different mutants of ELANE associated with SCN or to both CyN and SCN triggered different UPR pathways and suggested that SLPI may provide a protective function in some mutations by preventing the induction of the UPR [97]. SLPI is a reversible inhibitor of NE and CatG and is principally expressed in, and secreted by epithelial cells and it was also found in the cytoplasm of neutrophils [98]. It was later shown that SLPI is more likely localized in secondary granules of neutrophils as supported by expression pattern restricted to the myelocyte stage, subcellular co-localization, and secretion with lactoferrin [99]. Depending on the genotype, providing SLPI or another NE inhibitor at the right subcellular location may help reduce the severity of the neutropenia.

Lysosome/granule membrane permeabilisation. Experimentally, lysosomal or granule permeabilisation (GMP)-induced cell death has been studied using agents that have a detergent effect on membranes (Figure 1) [100]. L-leucyl L-leucine methyl ester (LLME) is a highly potent inducer of GMP in neutrophils. The mechanisms by which the LLME induces cell death require receptor-mediated endocytosis of LLME, which accumulates in granules where LLME is cleaved and polymerized into a detergent leucine polymers by DPPI $[101,102]$. Therefore, only cells that express DPPI can process, and are sensitive to LLME. However, strong bystander effects have been reported, where neutrophils and monocytes treated with LLME can induce apoptosis in NK and cytotoxic T cells that are not sensitive to micromolar concentrations of LLME [103]. Within neutrophils, all types of granules may not be similarly targeted since DPPI is found at higher levels in primary granules. Yet, since bystander cells can be sensitized to LLME, it is likely that all types of neutrophil granules are also permeabilised to some degree. In serpinb1-deficient neutrophils, CatG appears as an essential executioner of neutrophil death in LLMEtreated neutrophils, while NE has no direct impact on cell death even if it is stored in primary granules together with CatG [56]. Conversely, serpinb1 levels inversely correlate with neutrophil sensitivity to LLME [57].

Bacterial pathogens and their products can also interfere with GMP-mediated death. Type1-fimbriated $E$. coli and the $P$. aeruginosa toxin pyocyanin were shown to induce neutrophil apoptosis associated with ROS production, CatD activity, and caspase activation 
[104-106]. Pyocyanin induced the release of both CatD and CatG in the cytoplasm, but treatment with protease inhibitors suggested a prominent role for CatD in apoptosis [106].

Cell death associated with inflammation, death receptors and autophagy. Survival of neutrophils can be temporarily extended in vivo and in vitro by cytokines and growth factors, notably GM-CSF and G-CSF. Several mechanisms operate to delay neutrophil apoptosis such as transcriptional regulation of $\mathrm{Bcl}-2$ family proteins and reduced calpain activation [107-110]. Like other cell types, neutrophil apoptosis can be induced by ligation and clustering of death receptors of the TNF cytokine family, initiating the extrinsic pathway of apoptosis. While neutrophils express Fas, TNFR1, TNFR2 and TRAILR, neutrophils show a number of particularities depending on the context and the intensity of the stimulation, a subject that has been extensively reviewed [12,111]. It is worthwhile mentioning that TNF- $\alpha$ is an inflammatory cytokine and signaling through TNF receptors leads to NF-kB activation and the production of cytokines and chemokines. TNF- $\alpha$ was shown to trigger a range of pro- and anti-apoptotic effects. Particularly, TNFR1 was shown to induce apoptosis by the activation of p38 MAPK, class IA PI3Ks and reactive oxygen species (ROS) but did not require caspase-8 [112]. Neutrophils are sensitive to Fas ligation in vitro but Fas expression does not appear to regulate basal neutrophil life span in normal conditions in vivo [113]. However, Fas-mediated apoptosis occurs at inflammatory sites through interactions with cytotoxic lymphocytes and NK cells. In this context, Fas-induced death pathway is delayed/abrogated by signaling through pathogen associated molecular patterns (PAMPs) and DAMPs [114]. This type of complex regulation might allow dampening of inflammation in viral infection by inducing neutrophil apoptosis but yet allow a possibility for sustained neutrophil survival in case of secondary bacterial infection, which release PAMPs. Therapeutically interfering with death receptor pathways in infectious disease to reduce inflammatory damage may prove challenging as it may also adversely affect the innate response capacity mediated by neutrophils.

An additional level of regulation is provided by calpains following death receptor ligation. Calpain-1 and calpain-2 are ubiquitous non-lysosomal, non-caspase cysteine proteases, which require calcium for their activity. Calpains are in active form in the cytosol but they are maintained inactive by their specific endogenous inhibitor calpastatin. Upon Fas ligation, caspase-8 was shown to degrade calpastatin releasing active calpain1 , which was shown to activate the mitochondrial pathway through processing of Bax and ultimately caspase-3 activation [115]. Calpain-1 can also cleave autophagy-related protein 5 (ATG5), generating a truncated form of ATG5 able to trigger the mitochondrial apoptosis pathway [116]. Furthermore, a rise in intracellular $\mathrm{Ca}^{2+}$ concentration in apoptotic 
neutrophils was shown to induce a rapid transition to necrosis indicating that calpains may act also in the end stages of neutrophil death pathways [117].

Other receptors on neutrophil surface have emerged as new triggers of neutrophil death. Among them, sialic acid-binding immunoglobulin-like lectin (siglec)-9 ligation was shown to induce neutrophil death in a ROS-dependent manner [118]. This death pathway was enhanced in neutrophils of patients with sepsis or rheumatoid arthritis $[118,119]$. Moreover, siglec-9 mediated death featured a caspase-independent arm that was associated with cytoplasmic vacuolization reminiscent of autophagy [120]. Similarly, ligation of the hyaluronic acid receptor CD44 on the surface of GM-CSF primed neutrophils also induces non-apoptotic cell death which requires NADPH oxidasedependent ROS, but not caspases, and is associated with formation of large cytoplasmic vacuoles [24]. This type of cell death has recently been demonstrated to be RIPK3 and MLKL dependent [16]. Autophagy is primarily a pro-survival process that internally recycles nutrients from long-lived or misfolded proteins as well as intact or damaged organelles. Thus, the presence of autophagic vacuoles or autophagosomes during cell death should be viewed a failed effort by the cell to save itself from death while (or despite of) executing an autophagic process rather than death by autophagy. [121]. Autophagosomes have a characteristic double-membrane that ultimately fuse with lysosomes resulting in bulk degradation of the cargo. Deletion of ATG5 in mice demonstrated that neutrophil apoptosis regulation and neutrophil functions are not altered when autophagy is blocked [122]. In fact, ATG5-deficiency in neutrophils was associated with increased neutrophil progenitor proliferation and generally increased neutrophil numbers in the bone marrow, blood and secondary lymphoid organs. Conversely, blockade of p38 MAPK or mTORC1 induced autophagy in neutrophilic precursor cells and inhibited their differentiation indicating that autophagy is rather a negative regulator of granulopoiesis and has little impact on neutrophil apoptosis.

\section{Conclusion}

The cumulative evidence now indicates that neutrophil granule proteases should be considered as important regulators of the neutrophil life cycle and functions. Defects in these potent enzymes or their inhibitors are associated profound phenotypes that could be exploited to modulate neutrophil programmed cell death pathways. Further work should help define distinct molecular mechanisms leading to loss of granule integrity in neutrophil homeostasis and immune functions against pathogens. Understanding these process may have therapeutic applications in infectious, inflammatory and autoimmune diseases. 


\section{Acknowledgements}

CB is supported by grants of the Swiss National Science Foundation (grant no. 310030_149790), the Research Fund of the Swiss Lung Association, the OPO Stiftung and the Swiss Multiple Sclerosis Society. HUS is supported by grants of the Swiss National Science Foundation (grant no. 310030_166473), Swiss Cancer League (KFS3703-08-2015), HORIZON 2020, Marie Sklodowska-Curie Actions, MEL-PLEX, and Novartis Foundation for Medical-Biological Research.

\section{References}

[1] J.A. Lekstrom-Himes, J.I. Gallin, Immunodeficiency diseases caused by defects in phagocytes, N Engl J Med. 343 (2000) 1703-1714.

[2] P. Kruger, M. Saffarzadeh, A.N.R. Weber, N. Rieber, M. Radsak, H. von Bernuth, et al., Neutrophils: Between Host Defence, Immune Modulation, and Tissue Injury, PLoS Pathog. 11 (2015) e1004651.

[3] J. Pillay, T. Tak, V.M. Kamp, L. Koenderman, Immune suppression by neutrophils and granulocytic myeloid-derived suppressor cells: similarities and differences, Cell Mol Life Sci. 70 (2013) 3813-3827.

[4] W.M. Nauseef, N. Borregaard, Neutrophils at work, Nat Immunol. 15 (2014) 602-611.

[5] G.E. Cartwright, J.W. Athens, M.M. Wintrobe, The kinetics of granulopoiesis in normal man, Blood. 24 (1964) 780-803.

[6] J.T. Dancey, K.A. Deubelbeiss, L.A. Harker, C.A. Finch, Neutrophil kinetics in man, J Clin Invest. 58 (1976) 705-715.

[7] T.H. Price, G.S. Chatta, D.C. Dale, Effect of recombinant granulocyte colonystimulating factor on neutrophil kinetics in normal young and elderly humans, Blood. 88 (1996) 335-340.

[8] D.C. Dale, L.A. Boxer, W.C. Liles, The phagocytes: neutrophils and monocytes, Blood. 112 (2008) 935-945.

[9] J. Pillay, I. den Braber, N. Vrisekoop, L.M. Kwast, R.J. de Boer, J.A.M. Borghans, et al., In vivo labeling with $2 \mathrm{H} 2 \mathrm{O}$ reveals a human neutrophil lifespan of 5.4 days, Blood. 116 (2010) 625-627.

[10] K.W. Li, S.M. Turner, C.L. Emson, M.K. Hellerstein, D.C. Dale, Deuterium and neutrophil kinetics, Blood. 117 (2011) 6052-3.

[11] J. Lahoz-Beneytez, M. Elemans, Y. Zhang, R. Ahmed, A. Salam, M. Block, et al., Human neutrophil kinetics: modeling of stable isotope labeling data supports short blood neutrophil half-lives, Blood. 127 (2016) 3431-3438.

[12] B. Geering, H.U. Simon, Peculiarities of cell death mechanisms in neutrophils, Cell Death Differ. 18 (2011) 1457-1469.

[13] D. Wallach, T.-B. Kang, C.P. Dillon, D.R. Green, Programmed necrosis in inflammation: Toward identification of the effector molecules, Science. 352 (2016) aaf2154. 
[14] L. Sun, H. Wang, Z. Wang, S. He, S. Chen, D. Liao, et al., Mixed lineage kinase domain-like protein mediates necrosis signaling downstream of RIP3 kinase, Cell. 148 (2012) 213-227.

[15] M.C. Greenlee-Wacker, K.M. Rigby, S.D. Kobayashi, A.R. Porter, F.R. DeLeo, W.M. Nauseef, Phagocytosis of Staphylococcus aureus by human neutrophils prevents macrophage efferocytosis and induces programmed necrosis, $\mathrm{J}$ Immunol. 192 (2014) 4709-4717.

[16] X. Wang, Z. He, H. Liu, S. Yousefi, H.-U. Simon, Neutrophil necroptosis is triggered by ligation of adhesion molecules following GM-CSF priming, J Immunol. in press (2016).

[17] J. Shi, Y. Zhao, K. Wang, X. Shi, Y. Wang, H. Huang, et al., Cleavage of GSDMD by inflammatory caspases determines pyroptotic cell death, Nature. 526 (2015) 660-665.

[18] N. Kayagaki, I.B. Stowe, B.L. Lee, K. O'Rourke, K. Anderson, S. Warming, et al., Caspase-11 cleaves gasdermin $D$ for non-canonical inflammasome signalling, Nature. 526 (2015) 666-671.

[19] J. Ding, K. Wang, W. Liu, Y. She, Q. Sun, J. Shi, et al., Pore-forming activity and structural autoinhibition of the gasdermin family, Nature. 535 (2016) 111116.

[20] E.A. Miao, J.V. Rajan, A. Aderem, Caspase-1-induced pyroptotic cell death, Immunol Rev. 243 (2011) 206-214.

[21] K.S. Ravichandran, Beginnings of a good apoptotic meal: the find-me and eatme signaling pathways, Immunity. 35 (2011) 445-455.

[22] S. Aits, M. Jäättelä, Lysosomal cell death at a glance, J Cell Sci. 126 (2013) 1905-1912.

[23] U. Repnik, M. Hafner Česen, B. Turk, Lysosomal membrane permeabilization in cell death: concepts and challenges, Mitochondrion. 19 (2014) 49-57.

[24] C.C. Mihalache, S. Yousefi, S. Conus, P.M. Villiger, E.M. Schneider, H.-U. Simon, Inflammation-associated autophagy-related programmed necrotic death of human neutrophils characterized by organelle fusion events, J Immunol. 186 (2011) 6532-6542.

[25] K.M. Rigby, F.R. DeLeo, Neutrophils in innate host defense against Staphylococcus aureus infections, Semin Immunopathol. 34 (2012) 237-259.

[26] T.A. Fuchs, U. Abed, C. Goosmann, R. Hurwitz, I. Schulze, V. Wahn, et al., Novel cell death program leads to neutrophil extracellular traps, J Cell Biol. 176 (2007) 231-241.

[27] V. Brinkmann, U. Reichard, C. Goosmann, B. Fauler, Y. Uhlemann, D.S. Weiss, et al., Neutrophil extracellular traps kill bacteria, Science. 303 (2004) 15321535.

[28] D. Simon, H.U. Simon, S. Yousefi, Extracellular DNA traps in allergic, infectious, and autoimmune diseases, Allergy. 68 (2013) 409-416.

[29] S. Yousefi, H.-U. Simon, NETosis - Does It Really Represent Nature's "Suicide Bomber"? Front Immunol. 7 (2016) 328.

[30] S. Yousefi, C. Mihalache, E. Kozlowski, I. Schmid, H.U. Simon, Viable neutrophils release mitochondrial DNA to form neutrophil extracellular traps, Cell Death Differ. 16 (2009) 1438-1444.

[31] B.G. Yipp, B. Petri, D. Salina, C.N. Jenne, B.N.V. Scott, L.D. Zbytnuik, et al., Infection-induced NETosis is a dynamic process involving neutrophil multitasking in vivo, Nat Med. (2012).

[32] O.E. Sørensen, N. Borregaard, Neutrophil extracellular traps - the dark side of neutrophils, J Clin Invest. 126 (2016) 1612-1620.

[33] J.B. Cowland, N. Borregaard, Granulopoiesis and granules of human neutrophils, Immunol Rev. 273 (2016) 11-28.

[34] J.S. Savill, A.H. Wyllie, J.E. Henson, M.J. Walport, P.M. Henson, C. Haslett, Macrophage phagocytosis of aging neutrophils in inflammation. Programmed 
cell death in the neutrophil leads to its recognition by macrophages, J Clin Invest. 83 (1989) 865-875.

[35] S.M. Rankin, The bone marrow: a site of neutrophil clearance, J Leukoc Biol. 88 (2010) 241-251.

[36] S.J. Gardai, Y.-Q. Xiao, M. Dickinson, J.A. Nick, D.R. Voelker, K.E. Greene, et al., By binding SIRPalpha or calreticulin/CD91, lung collectins act as dual function surveillance molecules to suppress or enhance inflammation, Cell. 115 (2003) 13-23.

[37] R.W. Vandivier, P.M. Henson, I.S. Douglas, Burying the dead: the impact of failed apoptotic cell removal (efferocytosis) on chronic inflammatory lung disease, Chest. 129 (2006) 1673-1682.

[38] N. Thieblemont, H.L. Wright, S.W. Edwards, V. Witko-Sarsat, Human neutrophils in auto-immunity, Semin Immunol. 28 (2016) 159-173.

[39] M.A. Stark, Y. Huo, T.L. Burcin, M.A. Morris, T.S. Olson, K. Ley, Phagocytosis of apoptotic neutrophils regulates granulopoiesis via IL-23 and IL-17, Immunity. 22 (2005) 285-294.

[40] I. Jorgensen, Y. Zhang, B.A. Krantz, E.A. Miao, Pyroptosis triggers poreinduced intracellular traps (PITs) that capture bacteria and lead to their clearance by efferocytosis, J Exp Med. 213 (2016) 2113-2128.

[41] K. Theilgaard-Mönch, L.C. Jacobsen, R. Borup, T. Rasmussen, M.D. Bjerregaard, F.C. Nielsen, et al., The transcriptional program of terminal granulocytic differentiation, Blood. 105 (2005) 1785-1796.

[42] S. Rørvig, C. Honore, L.-I. Larsson, S. Ohlsson, C.C. Pedersen, L.C. Jacobsen, et al., Ficolin-1 is present in a highly mobilizable subset of human neutrophil granules and associates with the cell surface after stimulation with $f M L P, J$ Leukoc Biol. 86 (2009) 1439-1449.

[43] S. Rørvig, O. Ostergaard, N.H.H. Heegaard, N. Borregaard, Proteome profiling of human neutrophil granule subsets, secretory vesicles, and cell membrane: correlation with transcriptome profiling of neutrophil precursors, J Leukoc Biol. 94 (2013) 711-721.

[44] G. Lominadze, D.W. Powell, G.C. Luerman, A.J. Link, R.A. Ward, K.R. McLeish, Proteomic analysis of human neutrophil granules, Mol. Cell Proteomics. 4 (2005) 1503-1521.

[45] C.U. Niemann, M. Åbrink, G. Pejler, R.L. Fischer, E.I. Christensen, S.D. Knight, et al., Neutrophil elastase depends on serglycin proteoglycan for localization in granules, Blood. 109 (2007) 4478-4486.

[46] C.T.N. Pham, Neutrophil serine proteases: specific regulators of inflammation, Nat Rev Immunol. 6 (2006) 541-550.

[47] V. Witko-Sarsat, E.M. Cramer, C. Hieblot, J. Guichard, P. Nusbaum, S. Lopez, et al., Presence of proteinase 3 in secretory vesicles: evidence of a novel, highly mobilizable intracellular pool distinct from azurophil granules, Blood. 94 (1999) 2487-2496.

[48] M.J. McGuire, P.E. Lipsky, D.L. Thiele, Generation of active myeloid and lymphoid granule serine proteases requires processing by the granule thiol protease dipeptidyl peptidase I, J Biol Chem. 268 (1993) 2458-2467.

[49] R. Khokha, A. Murthy, A. Weiss, Metalloproteinases and their natural inhibitors in inflammation and immunity, Nat Rev Immunol. 13 (2013) 649-665.

[50] J.A. Huntington, R.J. Read, R.W. Carrell, Structure of a serpin-protease complex shows inhibition by deformation, Nature. 407 (2000) 923-926.

[51] A. Dubin, J. Travis, J.J. Enghild, J. Potempa, Equine leukocyte elastase inhibitor. Primary structure and identification as a thymosin-binding protein, $\mathrm{J}$ Biol Chem. 267 (1992) 6576-6583.

[52] E. Remold-O'Donnell, J. Chin, M. Alberts, Sequence and molecular characterization of human monocyte/neutrophil elastase inhibitor, Proc Natl Acad Sci USA. 89 (1992) 5635-5639. 
[53] C. Benarafa, J. Cooley, W. Zeng, P.I. Bird, E. Remold-O'Donnell, Characterization of four murine homologs of the human ov-serpin monocyte neutrophil elastase inhibitor MNEI (SERPINB1), J Biol Chem. 277 (2002) 42028-42033.

[54] C. Benarafa, T.E. LeCuyer, M. Baumann, J.M. Stolley, T.P. Cremona, E. Remold-O'Donnell, SerpinB1 protects the mature neutrophil reserve in the bone marrow, J Leukoc Biol. 90 (2011) 21-29.

[55] C. Benarafa, G.P. Priebe, E. Remold-O'Donnell, The neutrophil serine protease inhibitor serpinb1 preserves lung defense functions in Pseudomonas aeruginosa infection, J Exp Med. 204 (2007) 1901-1909.

[56] M. Baumann, C.T.N. Pham, C. Benarafa, SerpinB1 is critical for neutrophil survival through cell-autonomous inhibition of cathepsin G, Blood. 121 (2013) 3900-3907.

[57] S.S. Burgener, M. Baumann, P. Basilico, E. Remold-O'Donnell, I.P. Touw, C. Benarafa, Myeloid conditional deletion and transgenic models reveal a threshold for the neutrophil survival factor Serpinb1, Biol Chem. 397 (2016) 897-905.

[58] A.A. Belaaouaj, R. McCarthy, M. Baumann, Z. Gao, T.J. Ley, S.N. Abraham, et al., Mice lacking neutrophil elastase reveal impaired host defense against gram negative bacterial sepsis, Nat Med. 4 (1998) 615-618.

[59] A.M. Adkison, S.Z. Raptis, D.G. Kelley, C.T.N. Pham, Dipeptidyl peptidase I activates neutrophil-derived serine proteases and regulates the development of acute experimental arthritis, J Clin Invest. 109 (2002) 363-371.

[60] D.M. Maclvor, S.D. Shapiro, C.T.N. Pham, A.A. Belaaouaj, S.N. Abraham, T.J. Ley, Normal neutrophil function in cathepsin G-deficient mice, Blood. 94 (1999) 4282-4293.

[61] F. Loison, H. Zhu, K. Karatepe, A. Kasorn, P. Liu, K. Ye, et al., Proteinase 3dependent caspase-3 cleavage modulates neutrophil death and inflammation, $\mathrm{J}$ Clin Invest. 124 (2014) 4445-4458.

[62] F.L. Scott, C.E. Hirst, J. Sun, C.H. Bird, S.P. Bottomley, P.I. Bird, The intracellular serpin proteinase inhibitor 6 is expressed in monocytes and granulocytes and is a potent inhibitor of the azurophilic granule protease, cathepsin G, Blood. 93 (1999) 2089-2097.

[63] D. Kaiserman, S. Knaggs, K.L. Scarff, A. Gillard, G. Mirza, M. Cadman, et al., Comparison of human chromosome 6 p25 with mouse chromosome 13 reveals a greatly expanded ov-serpin gene repertoire in the mouse, Genomics. 79 (2002) 349-362.

[64] K.L. Scarff, K.S. Ung, H. Nandurkar, P.J. Crack, C.H. Bird, P.I. Bird, Targeted disruption of SPI3/Serpinb6 does not result in developmental or growth defects, leukocyte dysfunction, or susceptibility to stroke, Mol Cell Biol. 24 (2004) 40754082.

[65] E.C. Morris, T.R. Dafforn, S.L. Forsyth, M.A. Missen, A.J. Horvath, L. Hampson, et al., Murine serpin 2A is a redox-sensitive intracellular protein, Biochem J. 371 (2003) 165-173.

[66] A.J. Horvath, S.L. Forsyth, P.B. Coughlin, Expression patterns of murine antichymotrypsin-like genes reflect evolutionary divergence at the Serpina3 locus, J. Mol. Evol. 59 (2004) 488-497.

[67] N. Liu, S.M. Raja, F. Zazzeroni, S.S. Metkar, R. Shah, M. Zhang, et al., NFkappaB protects from the lysosomal pathway of cell death, Embo J. 22 (2003) 5313-5322.

[68] L. Li, S.M. Byrne, N. Rainville, S. Su, E. Jachimowicz, A. Aucher, et al., Brief report: serpin Spi2A as a novel modulator of hematopoietic progenitor cell formation, Stem Cells. 32 (2014) 2550-2556.

[69] M.L. Gabelloni, A.S. Trevani, J. Sabatté, J. Geffner, Mechanisms regulating neutrophil survival and cell death, Semin Immunopathol. 35 (2013) 423-437. 
[70] B. Fadeel, A. Ahlin, J.-I. Henter, S. Orrenius, M.B. Hampton, Involvement of caspases in neutrophil apoptosis: regulation by reactive oxygen species, Blood. 92 (1998) 4808-4818.

[71] N.A. Maianski, J. Geissler, S.M. Srinivasula, E.S. Alnemri, D. Roos, T.W. Kuijpers, Functional characterization of mitochondria in neutrophils: a role restricted to apoptosis, Cell Death Differ. 11 (2004) 143-153.

[72] B.M. Murphy, A.J. O'Neill, C. Adrain, R.W.G. Watson, S.J. Martin, The apoptosome pathway to caspase activation in primary human neutrophils exhibits dramatically reduced requirements for cytochrome C, J Exp Med. 197 (2003) 625-632.

[73] F. Altznauer, S. Martinelli, S. Yousefi, C. Thürig, I. Schmid, E.M. Conway, et al., Inflammation-associated cell cycle-independent block of apoptosis by survivin in terminally differentiated neutrophils, J Exp Med. 199 (2004) 1343-1354.

[74] V. Witko-Sarsat, M. Pederzoli-Ribeil, E. Hirsch, E. Hirsh, S. Sozzani, M.A. Cassatella, Regulating neutrophil apoptosis: new players enter the game, Trends Immunol. 32 (2011) 117-124.

[75] G. Maga, U. Hubscher, Proliferating cell nuclear antigen (PCNA): a dancer with many partners, J Cell Sci. 116 (2003) 3051-3060.

[76] V. Witko-Sarsat, J. Mocek, D. Bouayad, N. Tamassia, J.-A. Ribeil, C. Candalh, et al., Proliferating cell nuclear antigen acts as a cytoplasmic platform controlling human neutrophil survival, J Exp Med. 207 (2010) 2631-2645.

[77] A.G. Rossi, D.A. Sawatzky, A. Walker, C. Ward, T.A. Sheldrake, N.A. Riley, et al., Cyclin-dependent kinase inhibitors enhance the resolution of inflammation by promoting inflammatory cell apoptosis, Nat Med. 12 (2006) 1056-1064.

[78] V. Witko-Sarsat, S. Canteloup, S. Durant, C. Desdouets, R. Chabernaud, P. Lemarchand, et al., Cleavage of p21waf1 by proteinase-3, a myeloid-specific serine protease, potentiates cell proliferation, J Biol Chem. 277 (2002) 4733847347.

[79] U. Repnik, B. Turk, Lysosomal-mitochondrial cross-talk during cell death, Mitochondrion. 10 (2010) 662-669.

[80] S. Conus, R. Perozzo, T. Reinheckel, C. Peters, L. Scapozza, S. Yousefi, et al., Caspase- 8 is activated by cathepsin $D$ initiating neutrophil apoptosis during the resolution of inflammation, J Exp Med. 205 (2008) 685-698.

[81] S. Conus, C. Pop, S.J. Snipas, G.S. Salvesen, H.-U. Simon, Cathepsin D primes caspase-8 activation by multiple intra-chain proteolysis, J Biol Chem. 287 (2012) 21142-21151.

[82] Q. Zhou, G.S. Salvesen, Activation of pro-caspase-7 by serine proteases includes a non-canonical specificity, Biochem J. 324 (1997) 361-364.

[83] L. Wang, Q. Li, L. Wu, S. Liu, Y. Zhang, X. Yang, et al., Identification of SERPINB1 as a physiological inhibitor of human granzyme H, J Immunol. 190 (2013) 1319-1330.

[84] L. Hou, J. Cooley, R. Swanson, P.C. Ong, R.N. Pike, M. Bogyo, et al., The protease cathepsin $L$ regulates Th17 cell differentiation, J. Autoimmun. 65 (2015) 56-63.

[85] M. Germeshausen, S. Deerberg, Y. Peter, C. Reimer, C.P. Kratz, M. Ballmaier, The spectrum of ELANE mutations and their implications in severe congenital and cyclic neutropenia, Hum. Mutat. 34 (2013) 905-914.

[86] V. Makaryan, C. Zeidler, A.A. Bolyard, J. Skokowa, E. Rodger, M.L. Kelley, et al., The diversity of mutations and clinical outcomes for ELANE-associated neutropenia, Curr Opin Hematol. 22 (2015) 3-11.

[87] D.C. Dale, R.E. Person, A.A. Bolyard, A.G. Aprikyan, C. Bos, M.A. Bonilla, et al., Mutations in the gene encoding neutrophil elastase in congenital and cyclic neutropenia, Blood. 96 (2000) 2317-2322.

[88] D.C. Dale, D.C. Link, The many causes of severe congenital neutropenia, $N$ Engl J Med. 360 (2009) 3-5. 
[89] M. Horwitz, K.F. Benson, R.E. Person, A.G. Aprikyan, D.C. Dale, Mutations in ELA2, encoding neutrophil elastase, define a 21-day biological clock in cyclic haematopoiesis, Nat Genet. 23 (1999) 433-436.

[90] K. Boztug, P.M. Järvinen, E. Salzer, T. Racek, S. Mönch, W. Garncarz, et al., JAGN1 deficiency causes aberrant myeloid cell homeostasis and congenital neutropenia, Nat Genet. 46 (2014) 1021-1027.

[91] C. Klein, M. Grudzien, G. Appaswamy, M. Germeshausen, I. Sandrock, A.A. Schäffer, et al., HAX1 deficiency causes autosomal recessive severe congenital neutropenia (Kostmann disease), Nat Genet. 39 (2007) 86-92.

[92] C. Klein, Genetic defects in severe congenital neutropenia: emerging insights into life and death of human neutrophil granulocytes, Annu Rev Immunol. 29 (2011) 399-413.

[93] M.H. Albert, L.D. Notarangelo, H.D. Ochs, Clinical spectrum, pathophysiology and treatment of the Wiskott-Aldrich syndrome, Curr Opin Hematol. 18 (2011) 42-48.

[94] I. Köllner, B. Sodeik, S. Schreek, H. Heyn, N. von Neuhoff, M. Germeshausen, et al., Mutations in neutrophil elastase causing congenital neutropenia lead to cytoplasmic protein accumulation and induction of the unfolded protein response, Blood. 108 (2006) 493-500.

[95] D.S. Grenda, M. Murakami, J. Ghatak, J. Xia, L.A. Boxer, D.C. Dale, et al., Mutations of the ELA2 gene found in patients with severe congenital neutropenia induce the unfolded protein response and cellular apoptosis, Blood. 110 (2007) 4179-4187.

[96] O. Klimenkova, W. Ellerbeck, M. Klimiankou, M. Ünalan, S. Kandabarau, A. Gigina, et al., A lack of secretory leukocyte protease inhibitor (SLPI) causes defects in granulocytic differentiation, Blood. 123 (2014) 1239-1249.

[97] R. Nustede, M. Klimiankou, O. Klimenkova, I. Kuznetsova, C. Zeidler, K. Welte, et al., ELANE mutant-specific activation of different UPR pathways in congenital neutropenia, Br J Haematol. 172 (2016) 219-227.

[98] J.M. Sallenave, M. Si Tahar, G. Cox, M. Chignard, J. Gauldie, Secretory leukocyte proteinase inhibitor is a major leukocyte elastase inhibitor in human neutrophils, J Leukoc Biol. 61 (1997) 695-702.

[99] L.C. Jacobsen, O.E. Sørensen, J.B. Cowland, N. Borregaard, K. TheilgaardMönch, The secretory leukocyte protease inhibitor (SLPI) and the secondary granule protein lactoferrin are synthesized in myelocytes, colocalize in subcellular fractions of neutrophils, and are coreleased by activated neutrophils, J Leukoc Biol. 83 (2008) 1155-1164.

[100] S. Ivanova, U. Repnik, L. Bojic, A. Petelin, V. Turk, B. Turk, Lysosomes in apoptosis, Meth Enzymol. 442 (2008) 183-199.

[101] D.L. Thiele, P.E. Lipsky, Mechanism of L-leucyl-L-leucine methyl estermediated killing of cytotoxic lymphocytes: dependence on a lysosomal thiol protease, dipeptidyl peptidase I, that is enriched in these cells, Proc Natl Acad Sci USA. 87 (1990) 83-87.

[102] D.L. Thiele, P.E. Lipsky, The action of leucyl-leucine methyl ester on cytotoxic lymphocytes requires uptake by a novel dipeptide-specific facilitated transport system and dipeptidyl peptidase I-mediated conversion to membranolytic products, J Exp Med. 172 (1990) 183-194.

[103] D.L. Thiele, P.E. Lipsky, Regulation of cellular function by products of lysosomal enzyme activity: elimination of human natural killer cells by a dipeptide methyl ester generated from L-leucine methyl ester by monocytes or polymorphonuclear leukocytes, Proc Natl Acad Sci USA. 82 (1985) 2468-2472.

[104] R. Blomgran, L. Zheng, O. Stendahl, Uropathogenic Escherichia coli triggers oxygen-dependent apoptosis in human neutrophils through the cooperative effect of type 1 fimbriae and lipopolysaccharide, Infection and Immunity. 72 (2004) 4570-4578. 
[105] R. Blomgran, L. Zheng, O. Stendahl, Cathepsin-cleaved Bid promotes apoptosis in human neutrophils via oxidative stress-induced lysosomal membrane permeabilization, J Leukoc Biol. 81 (2007) 1213-1223.

[106] L.R. Prince, S.M. Bianchi, K.M. Vaughan, M.A. Bewley, H.M. Marriott, S.R. Walmsley, et al., Subversion of a lysosomal pathway regulating neutrophil apoptosis by a major bacterial toxin, pyocyanin, J Immunol. 180 (2008) 35023511.

[107] B. Dibbert, M. Weber, W.H. Nikolaizik, P. Vogt, M.H. Schöni, K. Blaser, et al., Cytokine-mediated Bax deficiency and consequent delayed neutrophil apoptosis: a general mechanism to accumulate effector cells in inflammation, Proc Natl Acad Sci USA. 96 (1999) 13330-13335.

[108] N.A. Maianski, D. Roos, T.W. Kuijpers, Bid truncation, bid/bax targeting to the mitochondria, and caspase activation associated with neutrophil apoptosis are inhibited by granulocyte colony-stimulating factor, J Immunol. 172 (2004) 70247030.

[109] N. Andina, S. Conus, E.M. Schneider, M.F. Fey, H.U. Simon, Induction of Bim limits cytokine-mediated prolonged survival of neutrophils, Cell Death Differ. 16 (2009) 1248-1255.

[110] B.J. van Raam, A. Drewniak, V. Groenewold, T.K. van den Berg, T.W. Kuijpers, Granulocyte colony-stimulating factor delays neutrophil apoptosis by inhibition of calpains upstream of caspase-3, Blood. 112 (2008) 2046-2054.

[111] B. Geering, C. Stoeckle, S. Conus, H.-U. Simon, Living and dying for inflammation: neutrophils, eosinophils, basophils, Trends Immunol. 34 (2013) 398-409.

[112] B. Geering, U. Gurzeler, E. Federzoni, T. Kaufmann, H.-U. Simon, A novel TNFR1-triggered apoptosis pathway mediated by class IA PI3Ks in neutrophils, Blood. 117 (2011) 5953-5962.

[113] B.A. Croker, J.A. O'Donnell, C.J. Nowell, D. Metcalf, G. Dewson, K.J. Campbell, et al., Fas-mediated neutrophil apoptosis is accelerated by Bid, Bak, and Bax and inhibited by Bcl-2 and Mcl-1, Proc Natl Acad Sci USA. 108 (2011) 1313513140.

[114] J.A. O'Donnell, C.L. Kennedy, M. Pellegrini, C.J. Nowell, J.-G. Zhang, L.A. O'Reilly, et al., Fas regulates neutrophil lifespan during viral and bacterial infection, J Leukoc Biol. 97 (2015) 321-326.

[115] F. Altznauer, S. Conus, A. Cavalli, G. Folkers, H.-U. Simon, Calpain-1 regulates Bax and subsequent Smac-dependent caspase-3 activation in neutrophil apoptosis, J Biol Chem. 279 (2004) 5947-5957.

[116] S. Yousefi, R. Perozzo, I. Schmid, A. Ziemiecki, T. Schaffner, L. Scapozza, et al., Calpain-mediated cleavage of Atg5 switches autophagy to apoptosis, Nat Cell Biol. 8 (2006) 1124-1132.

[117] R.J. Francis, S. Kotecha, M.B. Hallett, Ca2+ activation of cytosolic calpain induces the transition from apoptosis to necrosis in neutrophils with externalized phosphatidylserine, J Leukoc Biol. 93 (2013) 95-100.

[118] S. von Gunten, S. Yousefi, M. Seitz, S.M. Jakob, T. Schaffner, R. Seger, et al., Siglec-9 transduces apoptotic and nonapoptotic death signals into neutrophils depending on the proinflammatory cytokine environment, Blood. 106 (2005) 1423-1431.

[119] S. von Gunten, S.M. Jakob, B. Geering, J. Takala, H.-U. Simon, Different patterns of Siglec-9-mediated neutrophil death responses in septic shock, Shock. 32 (2009) 386-392.

[120] S. von Gunten, H.-U. Simon, Autophagic-like cell death in neutrophils induced by autoantibodies, Autophagy. 3 (2007) 67-68.

[121] G. Kroemer, B. Levine, Autophagic cell death: the story of a misnomer, Nat Rev Mol Cell Biol. 9 (2008) 1004-1010.

[122] S. Rožman, S. Yousefi, K. Oberson, T. Kaufmann, C. Benarafa, H.U. Simon, 
The generation of neutrophils in the bone marrow is controlled by autophagy, Cell Death Differ. 22 (2015) 445-456. 


\section{Figure legend}

Figure 1. Integration of granule proteases and serpinb1 in the principal modes of neutrophil PCD pathways. Cell-intrinsic factors are thought to regulate neutrophil survival in steady state in vivo through activation of executioner caspases. Cytoplasmic serpinb1 protects neutrophils from CatG- and PR3-mediated apoptosis by blocking activation of pro-caspases. In vitro, CatD can activate caspase-8 but there are no known endogenous inhibitors of CatD. In the absence of serpinb1, CatG can trigger neutrophil PCD independently of caspase activity. Rapid granule permeabilization by LLME leads to a rapid necrotic PCD mediated by CatG. 


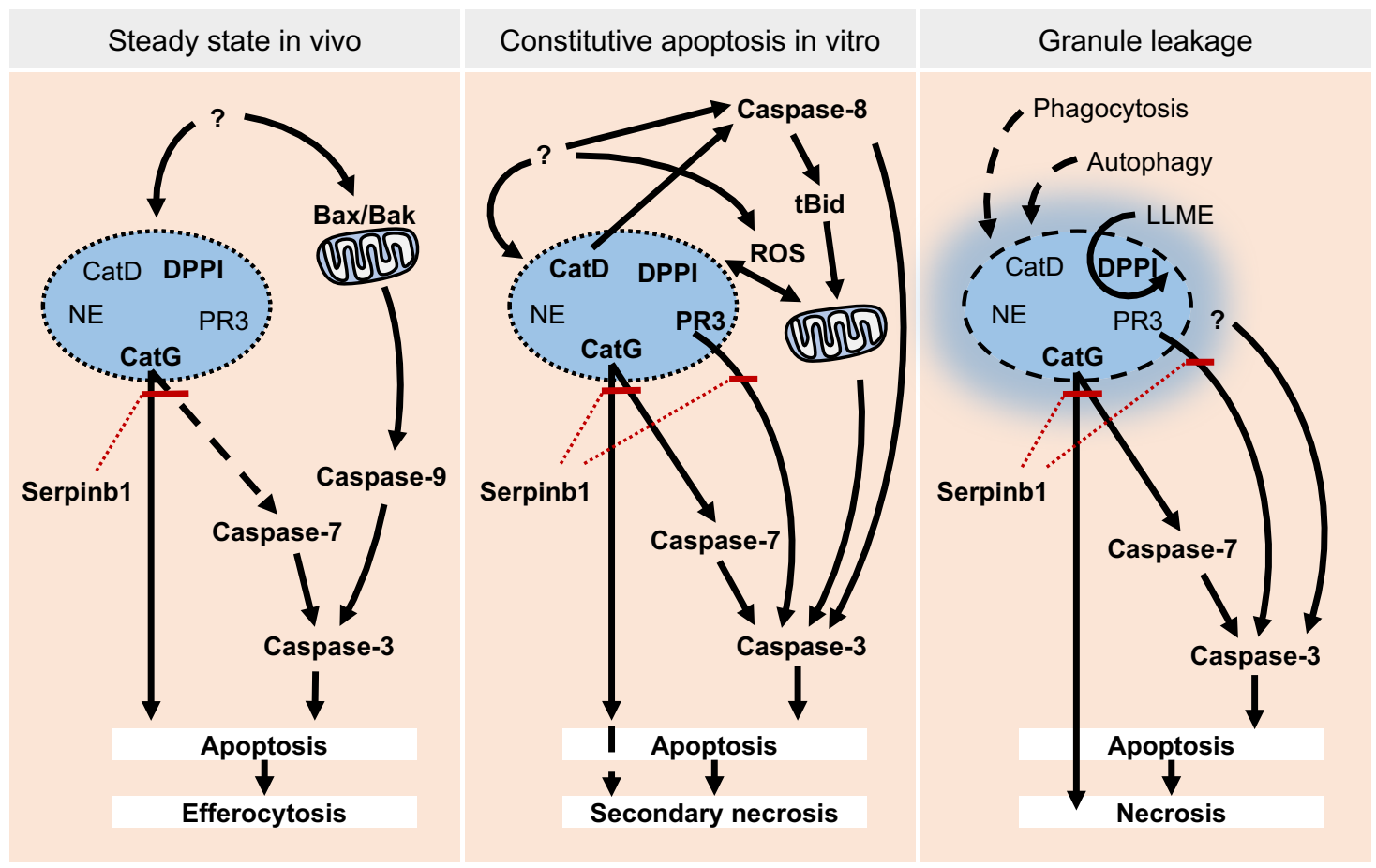

